Preprint typeset using LATEX style AASTeX6 v. 1.0

\title{
THE STELLAR DENSITY PROFILE OF THE DISTANT GALACTIC HALO
}

\author{
Colin T. Slater ${ }^{1}$, David L. Nidever ${ }^{2,3,4}$, Jeffrey A. Munn $^{5}$, Eric F. Belli ${ }^{4}$, Steven R. Majewski $^{6}$
}

\footnotetext{
${ }^{1}$ Department of Astronomy, University of Washington, Box 351580, Seattle, WA 98195, USA; ctslater@uw.edu

${ }^{2}$ Large Synoptic Survey Telescope, 950 North Cherry Ave, Tucson, AZ 85719; dnidever@lsst.org

${ }^{3}$ Steward Observatory, 933 North Cherry Ave, Tucson, AZ 85719

${ }^{4}$ Department of Astronomy, University of Michigan, 1085 S. University Ave., Ann Arbor, MI 48109;

${ }^{5}$ US Naval Observatory, Flagstaff Station, P.O. Box 1149, Flagstaff, AZ 86005

${ }^{6}$ Department of Astronomy, University of Virginia, Charlottesville, VA 22904
}

\section{ABSTRACT}

We use extensive gravity-sensitive DDO 51 photometry over 5100 square degrees, combined with SDSS broadband photometry, to select a catalog of $\sim 4,000$ giant stars covering a large fraction of the high Galactic latitude sky and reaching out to $\sim 80 \mathrm{kpc}$ in the Galactic halo. This sample of bright and unbiased tracers enables us to measure the radial profile and 3D structure of the stellar halo to large distance which had previously only been measured with sparse tracers or small samples. Using population synthesis models to reproduce the observed giant star luminosity function, we find that the halo maintains a $r^{-3.5}$ profile from 30 to $80 \mathrm{kpc}$ with no signs of a truncation or sharp break over this range. The radial profile measurement is largely insensitive to individual halo substructure components, but we find that attempting to measure the shape of the halo is overwhelmed by the Sagittarius stream such that no ellipsoidal shape is a satisfactory description in this region. These measurements allow us to begin placing the Milky Way in context with the growing sample of external galaxies where similar halo profile measurements are available, with the goal of further linking the properties of stellar halos to the accretion histories that formed them.

Keywords: Galaxy: halo, Galaxy: structure.

\section{INTRODUCTION}

The growth of stellar halos by accretion of satellite galaxies is a well- established feature of galaxy evolution. A wealth of individual structures are readily identifiable in large-scale surveys of the halo (e.g., Majewski et al. 2003; Belokurov et al. 2006; Slater et al. 2014), and statistical studies of fluctuations in number counts agree well with simulations of halos formed by numerous accretion events (Bell et al. 2008; Xue et al. 2011). While this general picture appears to hold, numerous details of how these accretion events combine to produce a halo are unclear. The radial profile and shape of the stellar halo are global quantities that should constrain halo formation models, since these parameters depend on the detailed process of how individual satellite accretions distribute their stars during the growth of the galaxy.

Extracting this information is complicated both on the observational and theoretical side by numerous difficulties. Observationally our most detailed measurements of the halo over wide areas of sky are often limited to volumes within $\sim 30 \mathrm{kpc}$ of the Sun, as most are dependent on using main sequence stars as tracers in surveys such as the Sloan Digital Sky Survey (SDSS) (Jurić et al. 2008; de Jong et al. 2010; Deason et al. 2011). Probing distances greater than this has required using deep pointings over a more limited area (Sesar et al. 2011), intrinsically brighter but sparse tracers such as RR Lyrae (Watkins et al. 2009; Sesar et al. 2010; Akhter et al. 2012; Cohen et al. 2015), or careful isolation of blue horizontal branch (BHB) stars from contamination by more populous foreground stars (Deason et al. 2014). These methods often suffer from either limited sky coverage, limited number counts, or potential contaminants which are difficult to remove. Additionally there is often the risk that the tracer population is not representative of the entire stellar mass at that radius, and that variations in stellar populations could produce a tracer profile that differs from the density profile.

In this work we introduce a large survey of DDO 51 photometry that aims to produce a well-characterized sample of giant stars in the stellar halo. The DDO 51 fil- 
ter has a narrow bandpass centered on the $\mathrm{MgH}$ and $\mathrm{Mg}$ b spectral features at $5100 \AA$, which exhibits a sensitivity to surface gravity (Clark \& McClure 1979). Geisler (1984) showed that the DDO 51 can be used with the broadband Washington filter system to measure surface gravity with the M and DDO 51 filters, along with temperatures via the $T_{1}$ and $T_{2}$ filters. This was further refined by Majewski et al. (2000), who found that $\mathrm{M}$ - $\mathrm{T}_{2}$ provided sufficient temperature information such that the $\mathrm{T}_{1}$ filter could be skipped, thus improving the observing efficiency.

In this work we realize another gain in observing efficiency by using the SDSS imaging for the broadband data, freeing our observing program to focus on collecting narrow band data. Through use of the robotic United States Naval Observatory (USNO) $1.3 \mathrm{~m}$ telescope we have obtained 5100 square degrees of imaging in DDO 51, yielding one of the largest samples of giants at distances of $30-80 \mathrm{kpc}$ in the Galactic halo. This enables us to measure the radial profile and shape of the halo with greatly reduced risk that small sample areas, varying stellar populations, or contamination are affecting our measurements.

Our effort focuses on measuring these bulk properties of the stellar halo, the radial profile and shape, by combining this dataset with synthetic stellar populations models that recreate the survey footprint and DDO 51 selection. We begin by discussing the DDO 51 data and our modeling of the resulting color- magnitude diagram (CMD) in Section 2. We discuss our synthetic modeling of the survey in Section 3 and our fits to the data in Section 4. We finish with a discussion of other halo profile measurements in Section 5 and our conclusions in Section 6.

\section{OBSERVATIONS}

All observations for our ongoing imaging survey are obtained using the Array Camera on the U.S. Naval Observatory, Flagstaff Station, 1.3 meter telescope. The Array Camera is a $2 \times 3$ mosaic of $2048 \times 4102 \mathrm{e} 2 \mathrm{v}$ CCDs with $0.6^{\prime \prime}$ pixels, and a field of view of $1.41^{\circ}$ in right ascension by $1.05^{\circ}$ in declination. Each field is observed with a single 1200 second exposure in the DDO51 filter. No standard star fields are observed during the night. Fields are separated by $1^{\circ}$ in declination, and roughly $1.34^{\circ}$ in right ascension (the separation in right ascension varies slightly with declination, so as to cover the entire $360^{\circ}$ along a given declination stripe with an integer number of evenly spaced fields). Observations are taken in dark time, within one week of new moon. The telescope is fully automated, and observations are obtained under a wide range of seeing and cloud conditions.

Observations commenced at the end of October 2011

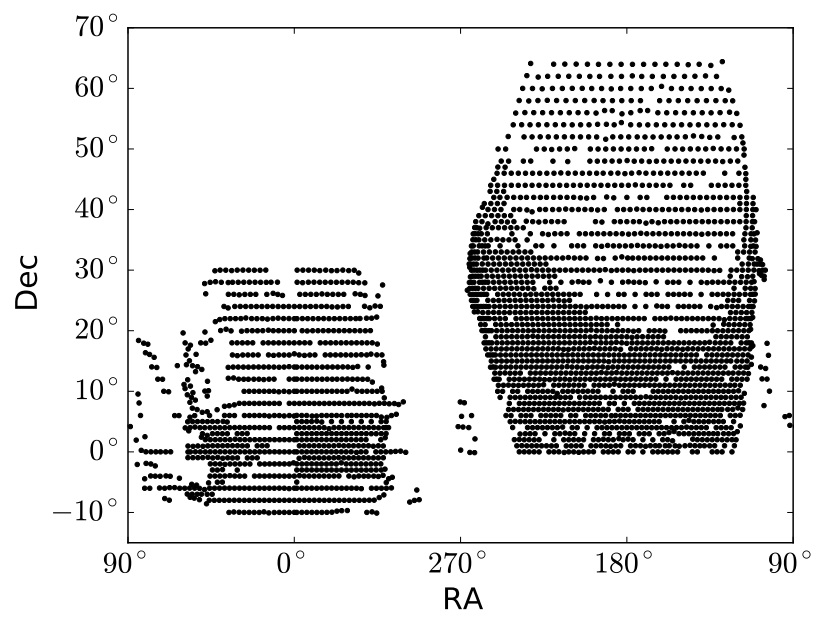

Figure 1. Sky coverage of the DDO51 observations. The survey follows the SDSS footprint so that broadband photometry can be used from that survey. In the northern Galactic hemisphere (right), the region at declination $<30^{\circ}$ is more densely filled simply due to the ongoing survey progress.

and are ongoing. The analysis in this work is based on data collected through June 2014, which includes 3,652 fields observed over 194 nights, for a total of $~ 5113$ square degrees. The sky coverage of observations is shown in Figure 1. The survey is designed to overlap the SDSS footprint so that broadband photometry is available for all fields. This results in a survey which is focused on high Galactic latitudes, roughly $|b|>30^{\circ}$. The observation pattern first sought to cover the SDSS footprint with a $25 \%$ filling factor, arranged into rows and columns in RA and Dec and skipping every other row and column. After that was completed, we began to fill in the remaining area, resulting in a denser coverage in some regions. The data currently cover approximately $40 \%$ of the target area.

Images are bias subtracted and flat-field corrected (using median twilight flats) using the Image Reduction and Analysis Facility (IRAF; Tody 1986, 1993) ${ }^{1}$. Object detection is performed using both SExtractor (Bertin \& Arnouts 1996) and DAOPHOT-II (Stetson 1987), which does a better job detecting stars near other bright stars. DAOPHOT-II is used to model the pointspread-function (PSF), which is allowed to vary quadratically with position in the frame, and to measure PSF and aperture magnitudes (using a $7.8^{\prime \prime}$ radius aperture) for each object. The PSF varies considerably across the wide field-of-view (FOV) of the telescope, introducing FOV dependent systematic differences between the PSF and aperture magnitudes. These are fit using a

\footnotetext{
${ }^{1}$ IRAF is distributed by the National Optical Astronomy Observatories, which are operated by the Association of Universities for Research in Astronomy, Inc., under cooperative agreement with the National Science Foundation.
} 
quadratic polynomial as a function of radial distance from the center of the FOV, correcting the PSF magnitudes to uncalibrated total magnitudes. The DDO51 data are then cross-matched with SDSS to provide gri colors.

To assure the best quality data for further analysis we apply the following cuts to the data on a chip-by-chip basis: $\mathrm{FWHM}<3.0^{\prime \prime}$, the photometric depth at which a signal to noise of 50 was reached must be fainter than 18th magnitude, and RMS scatter in the polynomial fit to the $7.8^{\prime \prime}$ radius aperture correction must be $<0.02$ mag. These criteria remove approximately $13 \%$ of all chips. All fits were also visually inspected (by D.L.N.) for systematic or pathological issues with the data or fits. This removed 205 fields (5\%), mostly at low-latitude.

The unique shape of the locus of dwarf stars ("swoosh") in the $g$-DDO51, $g-i$ diagram is used to calibrate the $g$ - DDO51 color produced from the instrumental, aperture corrected, PSF DDO51 magnitudes. We use the data from the entire survey to define a fiducial dwarf locus by finding the mean $g$ - DDO51 of the dwarfs as a function of $g-i$. Then to calibrate each chip, stars with $g<19.5$ are used determine the best (i.e., lowest $\chi^{2}$ ) constant offset in $g$-DDO51 that brings the dwarf stars into close alignment with our fiducial dwarf locus curve. This calibration procedure is similar to that used in Zasowski et al. (2013), who used similar data for target selection for the APOGEE survey. This curve is inherently specific to the survey, since small differences between DDO51 filters or between telescopes in which those filters are used could lead to a slightly different transmission curves and thus a differing dwarf locus. This does not impair our own analysis, but may limit direct numerical comparison to other surveys.

\section{CMD MODELING}

\subsection{Giant Selection}

For convenience we normalize out the "swoosh" feature using a polynomial fit to the ridgeline of the dwarf locus (where giants are negligible by number), over all fields. This produces the "adjusted" $g$ - DDO51 color seen in Figure 2, which we will refer to as $\Delta(g-$ DDO51). It should be noted that the exact form of dwarf locus in color-color space is sensitive to the particular filter's design and the telescope in which it is used (since they are interference filters), so specific values may be different in other DDO51 surveys.

In Figure 2 the giants lie in the feature that protrudes towards positive $\Delta(g-$ DDO51) values, starting at $g-i \sim 0.8$. The best separation between giants and dwarfs is achieved in the color range from roughly $1.2<g-i<1.6$, which corresponds to the upper half of the giant branch. Below that on the giant branch

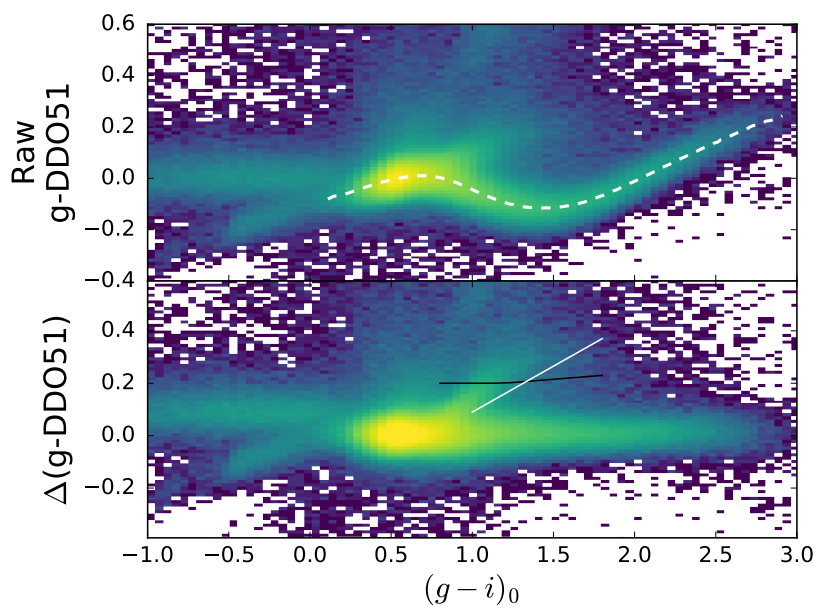

Figure 2. Color-color plots used for separating dwarfs from giants, showing all stars in the survey. The bulk of the density is from dwarfs, but a branch can be seen at $(g-i)_{0} \sim 1$ where the giants become distinct. The top panel shows the $g-$ DDO51 colors as observed, with the dashed line indicating the dwarf locus "swoosh", while in the bottom panel the g-DDO51 colors have been normalized such that the dwarfs are centered on $g-$ DDO51 $=0$. The giant locus can be seen above and parallel to the white line in the lower panel. The horizontal black line shows the color cut applied to select giants. Note that the separation between dwarfs and giants improves at redder colors, though there are intrinsically fewer giants towards the red.

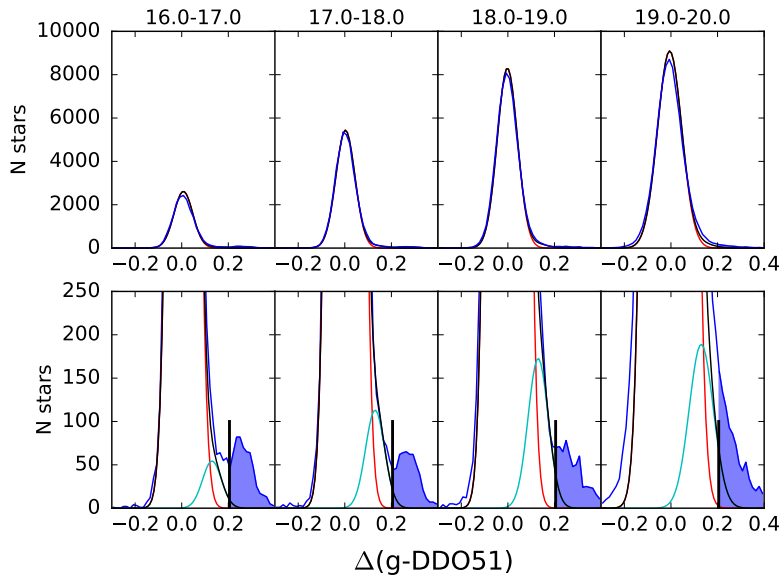

Figure 3. Histograms of $\Delta(g-$ DDO51)for stars with $1.2<$ $g-i<1.4$. The top panels show the full distribution in slices of apparent magnitude (bright to faint from left to right), while the bottom panels show the same points but with the y-axis rescaled to highlight the giant population. The observed data are in blue, our selection for giants is marked by the vertical black line and the filled blue area. A single Gaussian model of the dwarf contamination is shown in red, and a model with a second offset Gaussian (cyan) added is shown by the black model line. 


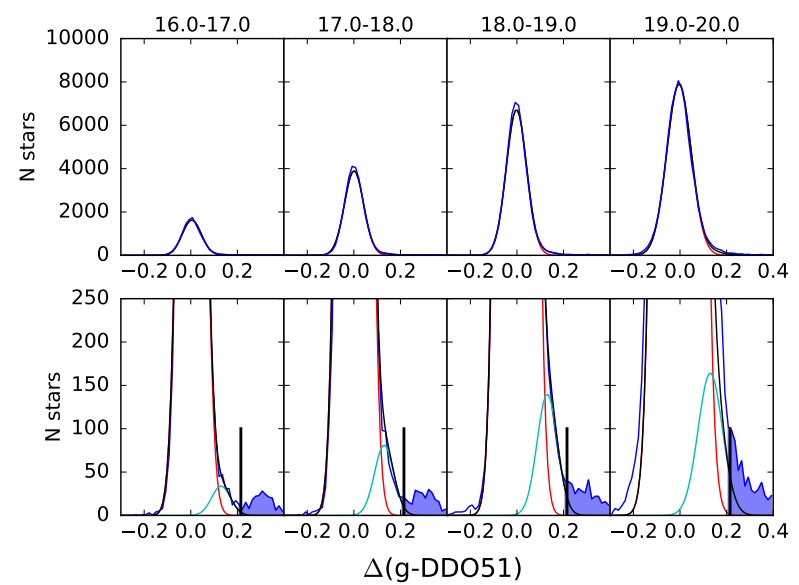

Figure 4. Same as Figure 3, but for stars with $1.4<g-i<$ 1.6. The separation between giants and dwarfs is slightly better, at the cost of diminished number counts.

(towards the blue in $g-i$ ) the gravity difference between giants and dwarfs is diminished. Focusing on this target color range, Figures 3 and 4 show histograms in $\Delta(g-$ DDO51) of stars with $1.2<g-i<1.4$ and $1.4<g-i<1.6$, respectively. The top row in these figures shows the full range of the data (blue lines), and it is immediately clear that the dwarfs dominate overwhelmingly, as expected. These panels also include a model of what we expect from the dwarf distribution in $\Delta(g-$ DDO51) assuming that the spread is the result of photometric uncertainty plus a small intrinsic scatter (Gaussian width $\sigma$ of $0.035 \mathrm{mag}$ ). For the bulk distribution of dwarfs this works extremely well, and there is very little deviation from a Gaussian.

Our goal, however, is to select the giants rather than modeling the dwarfs, and these can only be seen by zooming in on the tails of this distribution. The lower panels of Figures 3 and 4 are identical to the top panels but with a different y-axis scale to highlight the giants. This second peak in the data can be seen to the right of the dwarfs, and is highlighted with the blue shading. We define a $\Delta(g-$ DDO51) color cut (after removing the "swoosh") that falls in the valley between these dwarf and giant peaks, selecting everything to the right of that value as giants. We select as giants all sources with corrected $\Delta(g-\mathrm{DDO} 51)>0.20$ at $g-i<1.2$, and at $g-i>$ 1.2 we use $\Delta(g-$ DDO51) $>(0.20+0.05(\mathrm{~g}-\mathrm{i}-1.2))$. The slightly more aggressive selection takes advantage of the improved separation in $\Delta(g-$ DDO51) for redder giants. As will be discussed later, some dwarf contamination is inevitable with such a method, but our modeling will seek to account for that contamination in measuring the halo profile.

While the Gaussian fits to the dwarf distribution are overall excellent, in the zoomed-in panels of Figures 3 and 4 a small deviation between the data (blue) and the Gaussian model (red) can be seen. It is particularly important for us to understand the source of these excess stars, since a small fractional contamination by dwarfs could affect our inferred halo profiles unless properly modeled. Following extensive testing with SDSS spectroscopy, both with objects in common between datasets and with synthetic DDO51 photometry on SDSS spectra, it has become clear that this extension to higher $\Delta(g-$ DDO51 $)$ values is from dwarfs rather than any unique class of contaminants. It is expected that the $\Delta(g-$ DDO51) distribution of dwarfs should exhibit some variation with the metallicity of stars, since the measured equivalent widths can clearly be diminished by either lower gravity or lower abundance of metals. This effect is not negligible, but it is also of similar order to the photometric scatter, such that the dwarfs with slightly higher $\Delta(g-$ DDO51) colors are not ubiquitously low metallicity but are merely on average somewhat more likely to have lower metallicity. It is very challenging therefore to create a complete model of the metallicity distribution function for the disk dwarfs and their associated $\Delta(g-$ DDO51) distribution. Our solution is instead to model this heuristically. We approximate this complex metallicity function by the addition of a second Gaussian component centered on $\Delta(g-$ DDO51 $)=0.13$ and normalized to be $2 \%$ of the total number of dwarfs. These parameters are fixed across both color ranges and across magnitude bins. Figures 3 and 4 show this component in cyan, and the sum of it plus the main dwarf peak is the black line. The result matches the right-side slope of the dwarfs much better, without any tuning specific to particular magnitude bins (note we discard the $19<g<20$ magnitude bins in all fits). To be clear, we do not interpret this as an intrinsically distinct' component centered on $\Delta(g-\mathrm{DDO} 51)=0.13$, rather this pair of Gaussian functions is a suitable approximation to what is likely to be a convolution of a complex MDF with various uncertainties.

We also note that the metallicity of both the giants and dwarfs can affect their $g$ - DDO51 color, since the abundance of Magnesium influences the strength of the $\mathrm{Mg} \mathrm{b}$ and $\mathrm{MgH}$ spectral features. This metallicity dependence does not present a problem for our selection process because the lines are very weak in giants, and thus do not show as significant of a metallicity dependence as they do in dwarfs where the lines are strong. For dwarfs to reach the region in color-color space occupied by giants would require metallicities of $[\mathrm{Fe} / \mathrm{H}]=-3$ or less (see Figure 2 of Majewski et al. 2000), which is unlikely to occur in significant numbers. These aspects combine to limit the sensitivity of our selection process to the metallicity of either the giant or dwarf populations. 


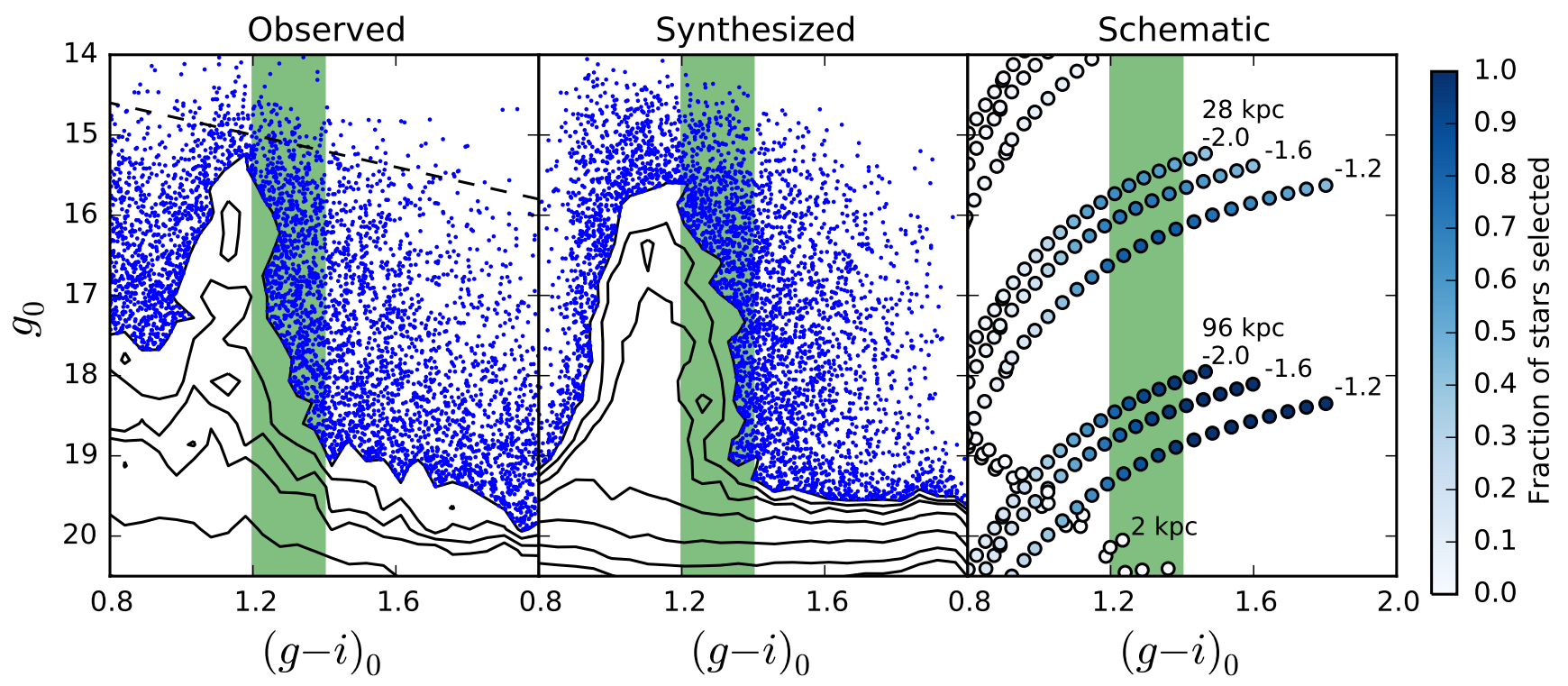

Figure 5. Observed CMD with the $\Delta(g-$ DDO51) selection for giants applied (left), along with the synthetic model CMD (center) and a schematic illustration of the isochrones that go into the model (right). High density regions have been replaced with contours for clarity, and the SDSS i-band saturation limit is shown by the horizontal dashed line. The primary color range of interest $\left(1.2<(g-i)_{0}<1.4\right)$ is show in green. A discussion of the general features of the CMD can be found in Section 3.3 . Overall the synthetic model reproduces the behavior of the observed CMD very well, including the rise of disk contamination at $g_{0}>19.5$ and the reduced dwarf-giant separation at $(g-i)_{0}<1.0$. The schematic diagram (right) shows the shape of the giant branch as it appears in our data, along with the distance and metallicity dependence (three metallicities at four distances are shown for clarity, though in the actual modeling we use 40 distance bins and 7 metallicity bins). The appearance of nearby dwarfs can also be seen; fractionally they are very well rejected from our sample, but their large numbers can cause significant contamination at $g_{0}>19.5$.

\subsection{CMD Modeling}

The resulting observed CMD of selected giants is shown in the left panel of Figure 5. Because the observed CMD is the result of a non- trivial selection process, combined with Galactic density profiles of the disk and halo, it can be difficult to interpret the resulting data by eye. We thus sought to better understand the observed data by creating a synthetic model of the CMD. This allows us to check our understanding of the relevant effects that give rise to the observations, along with the sensitivity of our results to changes in our assumed stellar populations, density distributions, and other sources of uncertainty.

Our modeling procedure is as follows. Each line of sight of the survey is treated independently, which are later combined in color-magnitude space to give a CMD of the entire survey. For each line of sight, a grid of isochrones are generated in both distance modulus and in metallicity (40 distance bins and 7 metallicity bins). For a given line of sight, any given distance modulus corresponds to a specific Cartesian position in the Galaxy, and we can use a Galactic density model (described below) to assign a total mass of stars we expect to be present in that volume. We then distribute that mass among the points on an isochrone from Dotter et al. (2008) according to a Kroupa (2001) initial mass func- tion.

Each point of each isochrone then corresponds to a total mass at a specific location in observed colormagnitude space. This is sufficient to determine the fraction of stars that would pass our selection criteria at each isochrone point, without having to generate large samples of artificial stars. Calculating the fraction of stellar mass that meets our $\Delta(g-$ DDO51) selection criteria can be done on the basis of an isochrone point's $g-i$ color and the photometric errors at that observed magnitude alone - a key assumption of our model is that dwarfs and giants live on specific loci in color-color space with only a Gaussian spread (largely due to photometric uncertainty). This assumption works remarkably well, and allows us to compute the fraction of stars that meet our color cut via the simple use of error functions. That is, for each point on each isochrone, we can compute the fraction that will fall into our $g-i$ selection window given the observed level of scatter, then which fraction will also meet our $\Delta(g-$ DDO51) selection cut.

This is then summed over all fields to yield the model luminosity function, or without the selection cuts, can be sampled to produce a simulated CMD. The resulting CMDs trace the increasing contamination at faint magnitudes along with its dependence on $g-i$ color, as we will discuss below. This process is repeated for each 
step in our metallicity grid independently, and the results summed according to the modeled density in that metallicity bin. Other stellar populations parameters are held fixed, though variation in them could also be implemented at higher computational cost.

We parameterize the density profile of the halo as a broken power law, where both the inner and outer power law slopes along with the break radius are potentially variable in the fitting process (Section 4). This is the only density component that is needed in the modeling process; the contamination from the Galactic disk dwarfs is measured directly in the observations themselves (as described at the end of Section 3.1). The contamination from disk giants is assumed to be negligible over our distance range of 30 to $80 \mathrm{kpc}$.

\subsection{Qualitative CMD Properties}

The resulting synthetic CMD can be seen in the center panel of Figure 5, along with a schematic illustration in the right panel. Overall, the qualitative level of agreement with the observed CMD is excellent, and confirms that we properly understand the relevant observational effects that play a role in generating the data. At bright magnitudes $(g<19)$, the selection of giants vastly dominates over dwarfs. The central density peak at $g-i \sim 1.1$ is caused by two competing factors: redwards of this peak the number of observed giants is decreasing because the intrinsic number towards the tip of the giant branch is decreasing, while bluewards of the peak the separation between giants and dwarfs in $\Delta(g-$ DDO51) becomes poorer and fewer giants are selected observationally. The exact position of this peak and its bluewards drop-off are thus sensitive to observational factors, and we avoid basing our conclusions on this region. Towards the red, the selection is much cleaner, which yields an ideal portion of the CMD from which to measure the distant density profile of the halo. As we will show below, we make use of two color ranges, $1.2<g-i<1.4$ and $1.4<g-i<1.6$, to further check that our profile measurements are robust. While the $1.2-1.4$ color range provides better statistics due to the greater number of stars, the $1.4-1.6$ color range has a different dependence on distance and on contamination, thus providing a double-check of our inferences.

At faint magnitudes the rise in disk contamination can be clearly seen (inside much of the contoured region in Figure 5 at $g=19$ and fainter). This also exhibits a color dependence; as the dwarf-giant separation increases towards the red, our color-cut also becomes slightly more aggressive in rejecting dwarfs. The number of contaminating dwarfs is thus reduced, and they do not begin to match the number of selected giants until fainter in the CMD. Finally, at the bright end of the CMD we are limited by saturation of the SDSS i-band data.

To match the color distribution of the observed CMD it is also necessary to include a spread of metallicities rather than a single stellar population. As can be seen in the schematic isochrones of Figure 5, if, for example, we created a synthetic CMD with only $[\mathrm{Fe} / \mathrm{H}]=-2.0$, there would be no giants redwards of $g-i=1.5$. That would be obviously inconsistent with the observations. Similarly, if we used only a higher metallicity isochrone, the distribution of stars in color would be far more uniform than is observed in the CMD, where a gradual taper in density from $g-i=1.2$ to 1.8 is observed. Reproducing this requires multiple isochrones of varying metallicity. Adopting a Gaussian metallicity distribution function (MDF) of width 0.4 dex centered between roughly $[\mathrm{Fe} / \mathrm{H}]=-1.5$ and -1.8 satisfies these qualitative requirements and is consistent with MDF measurements in SDSS by An et al. (2013). Figure 5 shows that metal-poor giants are preferentially brighter than metal-rich giants, and since our measurement range is fixed in apparent magnitude we measure slightly further if the halo is more metal-poor. At a metallicity of $[\mathrm{Fe} / \mathrm{H}]=-1.5$ giants at $g_{0}=18.5$ correspond to a heliocentric distance of $86 \mathrm{kpc}$, while $[\mathrm{Fe} / \mathrm{H}]=-1.3$ and -1.7 correspond to 77 and $95 \mathrm{kpc}$ respectively. In our fitting of the halo profile the MDF center is a free parameter, and a further discussion of the impact of the MDF on the inferred profile can be found in Section 4.2.

\section{HALO RADIAL PROFILE}

Once we have confirmed that we are able to accurately create synthetic CMDs for a given density model, we can begin to infer the best fitting spherical halo profile. An illustration of various halo outer slopes is shown in Figure 6, with the observed data shown by the thick blue stepped line. The estimated contamination from dwarfs is shown by the thin black line. In each case we have normalized the model LF to match the observed data. The upturn in counts fainter than 18.5 is caused by contamination from disk dwarfs. Brighter than $g_{0}=$ 16 photometric saturation in the SDSS data also limits our observed counts, which is also modeled but should not be treated as reliable.

Excluding these more uncertain regions, from $g_{0}=16$ to $g_{0}=18.5$ the data show a relatively flat distribution with magnitude. While such a flat distribution corresponds to an $r^{-3}$ density profile, in this case the small but growing contamination at fainter magnitudes implies a slightly steeper halo density profile of $r^{-3.5}$ after accounting for contamination. This is readily reproduced by models with an $r^{-3.5}$ density profile, as can be seen by eye. Models with steeper or shallower profiles at the \pm 0.5 level are clearly discrepant with the data, and there is no evidence of a significant density break beyond 


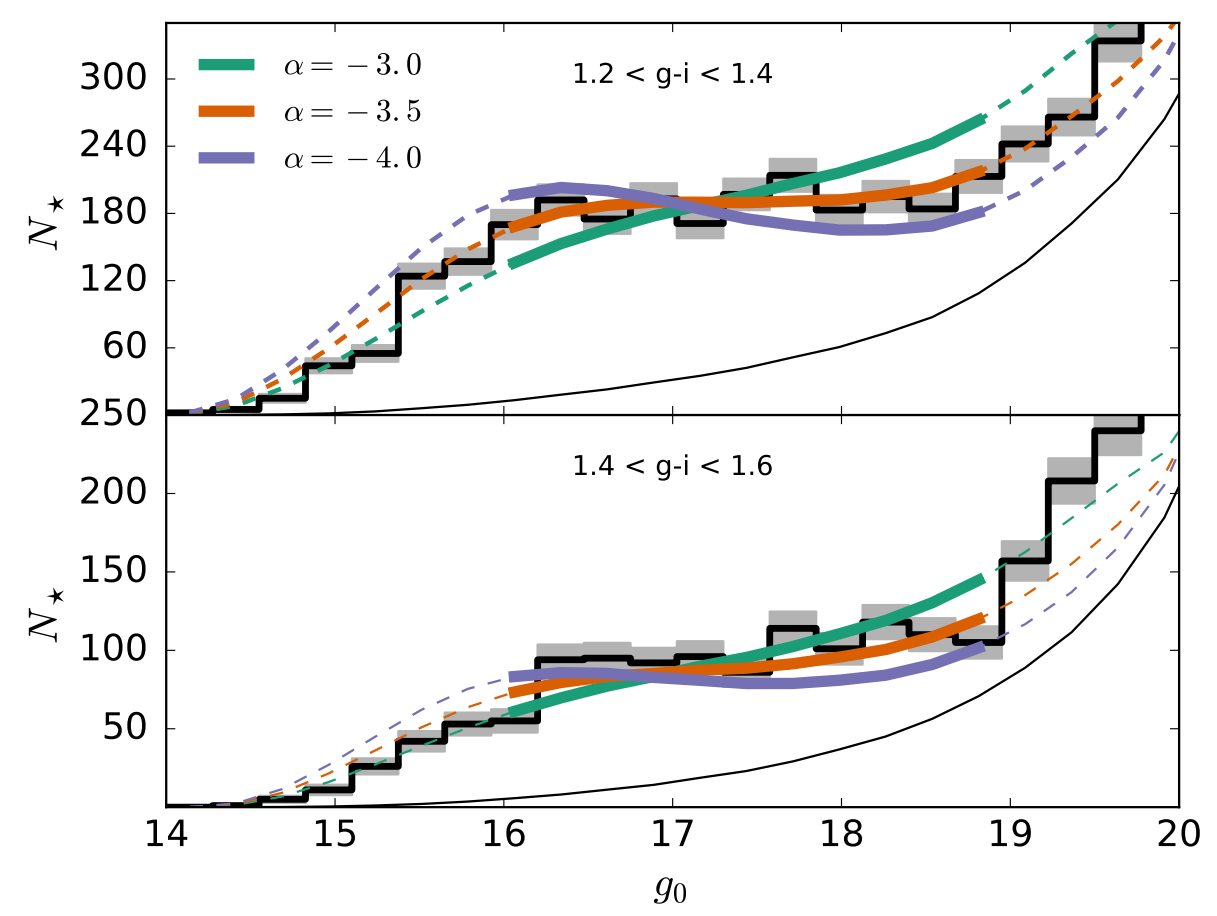

Figure 6. Comparison of different outer halo slopes. In each case the halo has a fixed $r^{-3.0}$ profile out to $27 \mathrm{kpc}$, at which point differing power law slopes $(\alpha)$ were adopted. Each model halo was then adjusted in normalization to match the observed $1.2<g-i<1.4 \mathrm{LF}$, and that normalization was carried over to the $1.4<g-i<1.6$ LF. Portions of the model outside the region for fitting are shown as dashed lines, and the thin black line shows our estimated dwarf contamination. A slope of $\alpha=-3.5$ reproduces the observations well, while steep slopes of $\alpha \leq-4.0$ are strongly discrepant.

$30 \mathrm{kpc}$. This is true both in the $1.2<(g-i)_{0}<1.4$ color bin and in $1.4<(g-i)_{0}<1.6$. With this redder color bin we have not renormalized any of the model profiles to the redder data; the normalization is carried over from the match to the blue color bin. The success of our density models in reproducing both LFs simultaneously provides an additional check that our modeling is reliable.

\subsection{MCMC Fits}

To follow up this visual comparison with a quantitative measurement, we combine our model LF generation process with the Markov Chain Monte Carlo code emcee (Foreman-Mackey et al. 2013). We compare our synthetic LFs with the data by computing the Poisson likelihood that the observed LF was drawn from the model. Our halo density model parameters include an inner slope, a break radius, and an outer slope, although we have limited sensitivity to the inner portion of this profile. We include these components for the purposes of marginalizing over them, ensuring that our outer slope measurement is not biased or overly precise due to our incomplete data at the bright end. We choose to only compare the luminosity functions where the data are mostly free from bright-end saturation issues and from faint-end disk contamination, using only the regions $16.0<g_{0}<18.5$. Both the inner slope and the outer slope were constrained in the MCMC sampling to values between -2.0 and -4.0 , since by visual inspection the outer slope was clearly well within this range and literature measurements of the inner slope had found similar values (See Table 5 in Akhter et al. 2012, for reference). The break radius was constrained to within 15 and $45 \mathrm{kpc}$, again bracketing the range of measurements from previous works. The addition of a Gaussian prior on the break radius had little impact on the resulting outer slope measurement except to reduce the uncertainties. The halo MDF was kept constant in the fit, since measuring the MDF is beyond the scope of this work.

The resulting measured outer slope value is $\alpha=$ $-3.5 \pm 0.2$, which is clearly in agreement with the visual comparison in Figure 6. There is very little covariance between the outer slope and the inner slope or break radius; our data simply do not probe these regions sufficiently.

The marginalized probability distribution functions for the halo shape parameters can be seen in Figure 7 . While the outer slope is well-constrained, the inner slope fit permits a wide range of values since we have little data in this region. The break radius is only slightly more constrained than the imposed prior (shown in orange). We also show the covariance between the outer slope measurement and the halo metallicity center. 

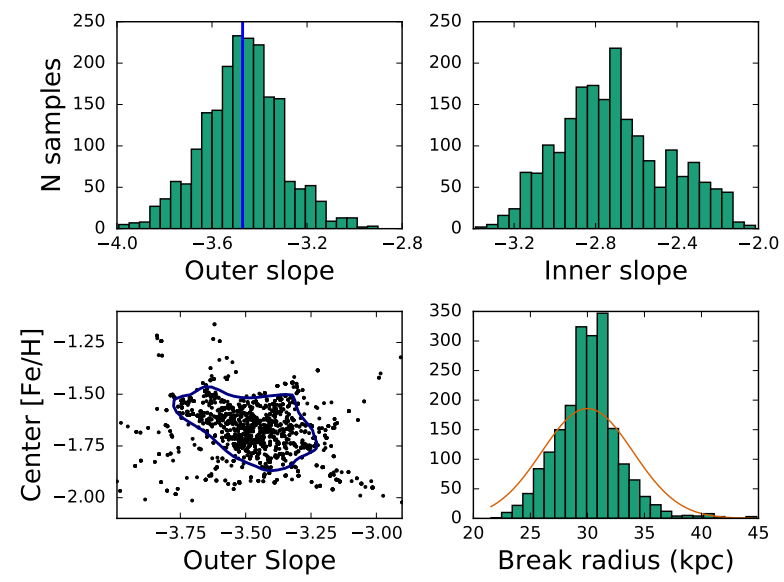

Figure 7. Marginalized probability distribution functions for three shape parameters, and MCMC samples showing the covariance between metallicity and outer halo slope (bottom left, $1 \sigma$ area outlined in blue). The break radius prior is shown in orange, while the two slopes had flat priors. The break radius is not strongly constrained by this fit.

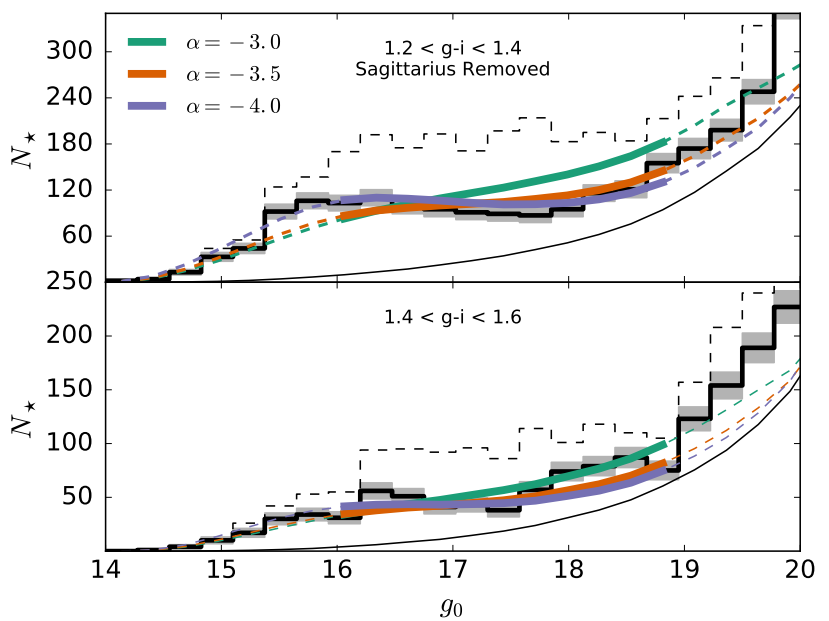

Figure 8. Observed luminosity functions with all fields within $12^{\circ}$ of the Sagittarius plane removed. The original LF is shown as the dashed blue histogram, and range of model slopes are overplotted (similar to Figure 6). The Sagittarius stream is not driving our halo radial profile measurement.

The interpretation of a single radial profile as having broad significance for the entire halo is complicated by the existence of, and likely dominance of, accreted and poorly-mixed substructure in the halo. This presents the question of whether one is able to measure a halo-wide quantity based on a limited number of pointings with varying degrees of identifiable substructures present in each? Clearly we would not put much confidence that a single line of sight through the halo, which would result in a radial profile which rises and falls as it passes through various structures, would be strongly representative of the entire halo. Our wide survey footprint is well-suited to avoiding such an effect, but there remains

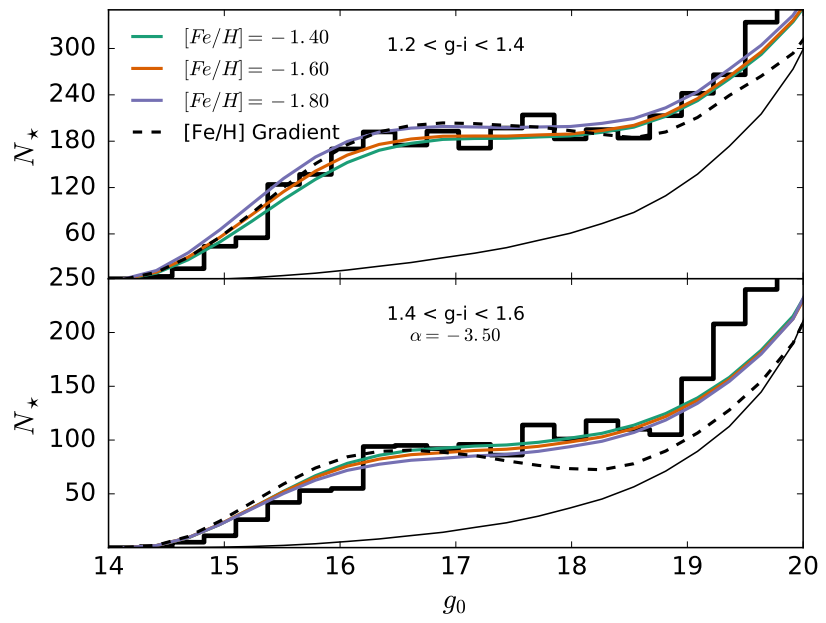

Figure 9. Metallicity dependence of the synthetic LF, for both color selections. Each model has an $r^{-3.5}$ outer density slope but different mean metallicities. Different metallicities impact the slope slightly due to the different distance ranges they cover in the halo. More notably, different assumed metallicities impact the relative counts between the two color bins, as a more metal-poor halo tends to push stars into the bluer color bin. An artificially steep metallicity gradient is also shown by the dashed black line, which strongly disagrees with the data.

a risk that, because the Sagittarius stream covers a large fraction of this footprint, our radial profile may be more a measurement of Sagittarius than of the halo.

To mitigate this concern, we show in Figure 8 a luminosity function where all fields within $\pm 12^{\circ}$ of the Sagittarius plane (as defined by Majewski et al. 2003) are excised from the data. The resulting contribution of the halo relative to the disk is reduced due to the decreased coverage at high Galactic latitudes, but the inferred radial profile is essentially unchanged. In part this is due to the mass of Sagittarius not dominating the total mass of the halo over the region in which we are sensitive, and is also a result of the significant distance variation that Sagittarius exhibits; it spreads material across the LF rather than piling up at at a single distance. Performing our MCMC fits on the Sagittariusexcised data produces a halo slope of $\alpha=-3.5 \pm 0.2$, indistinguishable from the fit with the entire dataset. Because Sagittarius is the most dominant known halo substructure, we argue that our measured radial profile is not dominated by any single structure, and can be meaningfully (but imperfectly) representative of the halo in bulk.

\subsection{Metallicity Effects}

Our analysis also depends on the metallicity distribution function (MDF) for the halo. As discussed above, the metallicity of the halo determines what portion of the giant branch falls into our selected color cuts, and also sets the distances we infer for these stars. We dis- 
cussed above that a broad MDF is required both by studies of the local halo stars and by the color distribution of giants in our sample. However, the center of this MDF is uncertain, particularly at the large distances probed by our sample of giants. Varying the MDF has two primary effects on our models: a different metallicity profile changes where the $\sim 30 \mathrm{kpc}$ density break appears in magnitude space, and it also changes the relative number of counts between our two color bins. These effects can both be seen in Figure 9. To accommodate these effects we have left the center of the MDF as a free parameter in the MCMC fitting, with a fixed width of 0.4 dex. The resulting metallicity preferred by the fit is $[\mathrm{Fe} / \mathrm{H}]=-1.7 \pm 0.14$. This falls in between the halo metallicity reported by An et al. (2013) of their "coadd sample", $[\mathrm{Fe} / \mathrm{H}]=-1.5$, and their "calibration sample", $[\mathrm{Fe} / \mathrm{H}]=-1.8$. The value from our fit is reasonable given the state of independent measures of the halo metallicity. The impact of this metallicity fit on our inferred halo profile is shown in the lower left panel of Figure 7. While changing halo metallicity can drive the outer slope measurement by a few tenths, any metallicity that can reasonably fit the fraction of stars in the two color bins still produces a halo profile slope between -3.75 and -3.25 .

A scenario which does potentially present a greater impact on our inferred profiles is that of a halo metallicity gradient. In this case a different portion of the giant branch at each distance will fall into our color cut, and can alter the observed number counts. While some previous studies, such as de Jong et al. (2010), have found decreasing metallicities between 10 and $30 \mathrm{kpc}$ from the sun, other studies have argued for a constant metallicity in this region (e.g. Ivezić et al. 2008). In either case, we can evaluate the effect that such a scenario would have on our inferred density profiles. To do this we created a toy metallicity gradient model, where the MDF center changes linearly from $[\mathrm{Fe} / \mathrm{H}]=-1.3$ at $20 \mathrm{kpc}$ (Galactocentric) to $[\mathrm{Fe} / \mathrm{H}]=-2.0$ at $100 \mathrm{kpc}$. The MDF width of 0.4 dex is preserved at all radii. The result of this model can be seen in Figure 9 by the dashed black line, where the underlying density profile is $\alpha=-3.5$. The result is an artificially steep drop in the LF, despite having the same density profile. A negative metallicity gradient would cause us to infer an artificially steep density profile. This can be understood intuitively by comparison to the isochrones in Figure 5. At bright magnitudes the high metallicity isochrone dominates, and the $1.2<g-i<1.4$ color cut samples relatively faint on the giant branch. Moving to larger distances and lower metallicities puts the top of the giant branch into the color cut, which has significantly fewer stars due to the intrinsic steepness of the giant branch luminosity function.
It is clear then that only a positive metallicity gradient would cause us to erroneously infer a shallower density profile than was actually present, but there is presently no evidence for such a gradient in the halo.

\subsection{Halo Oblateness}

In addition to the radial profile, the shape of the stellar halo as a whole may also hold information about the formation mechanisms of the halo and potentially about the accretion history of the Milky Way. This is particularly true in light of the suggestion that some component of the halo may be the result of "kicked up" Galactic disk stars, which are scattered to large heights by mergers (Zolotov et al. 2009; Purcell et al. 2010; Font et al. 2011). Such stars may leave a signature in the resulting shape of halo, as one might expect a flattened distribution of stars if their orbits have not been completely randomized. In models such as these the kickedup component is only significant in the inner $10-30 \mathrm{kpc}$ of the halo, and distances beyond this are dominated by accreted stars. Our data sample this outer region where the kicked up disk star fraction is likely to be negligible, thus a shape measurement at these distances presents an informative comparison to similar measurements of the inner regions of the halo.

Our procedure for measuring the halo shape is to construct model LFs for each telescope pointing individually, rather than a single LF for the sum total of the survey. These individual LFs will vary in their overall level depending on their line of sight through their halo. An illustration of the magnitude we expect to see is shown in Figure 10, which shows the ratio of counts expected in a model with $q=0.6$ over a spherical model. As expected, this ratio is mostly dependent on Galactic latitude, and much of the information in the measurement comes from low latitude fields towards the Galactic anticenter and in the southern Galactic hemisphere. It is important to note that the shape of the luminosity function does not change with varying oblateness; an $r^{-3}$ halo still produces a constant LF with magnitude regardless of flattening. For this reason we use only our best fit radial profile from Section 4 but with varying flattening factors. Changing the flattening of a model halo at fixed normalization also changes the total number of stars it predicts in our survey, hence in discussing non-spherical models we also adjust the normalization so that it matches in number the overall LF.

A visualization of the halo shape is shown in Figure 11, showing the surface density of giants against Galactic latitude. Model halos are also shown for comparison, both flattened, spherical, and prolate. It is clear that no ellipsoidal halo model is a suitable fit. There is enhancement in density at both low and high latitudes with a depression in between, which demands a degree 

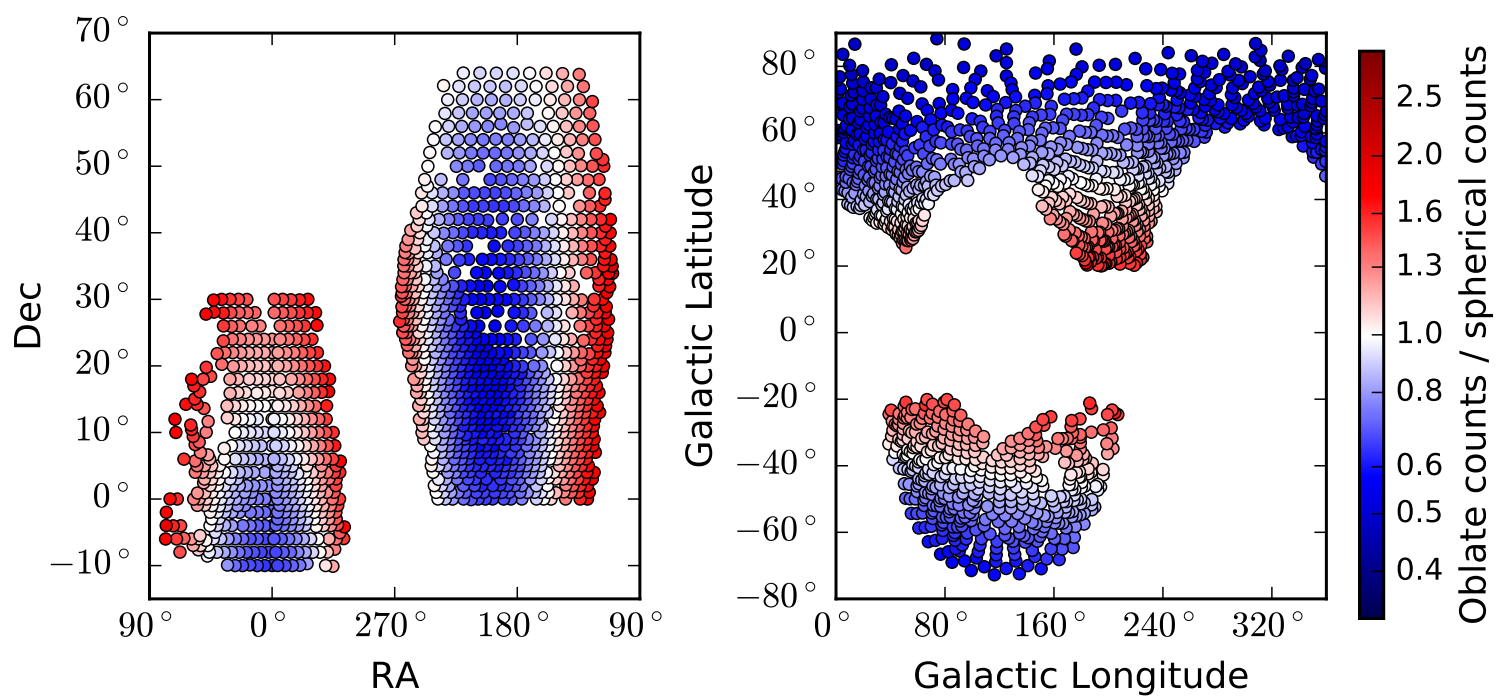

Figure 10. Ratio of counts in a $q=0.6$ flattened model to a spherical model, shown in equatorial coordinates on the left and Galactic coordinates on the right. This illustrates the form of the effect we would see in a non-spherical halo, with mostly a dependence on Galactic latitude but also slightly modulated by Galactic longitude (since the Sun is not in the center of the Galaxy).

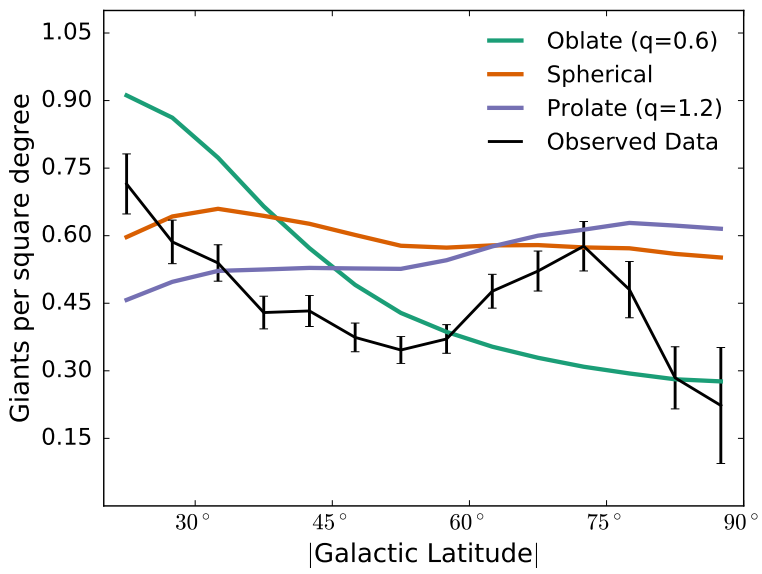

Figure 11. Visualization of the halo shape measurement. The mean number of giants per square degree of surveyed area is computed (black), and compared to the expectations for flattened ( $q=0.6$, blue) and prolate $(q=1.2$, red $)$ models and a spherical halo model (green line). The data exhibit an excess at both high and low latitudes such that no ellipsoidal shape could be considered a good fit.

of freedom not allowed by either oblate or prolate models. Attempting to fit this with such a model would not produce any acceptable summary of the halo's shape.

As can be seen qualitatively in images like the "Field of Streams" (Belokurov et al. 2006) and quantitatively in measurements such as Bell et al. (2008), Starkenburg et al. (2009), and Xue et al. (2011), the halo contains significant density substructure which may make spherical or ellipsoidal models poor fits to the data. To illustrate the effect of substructure in our data, Figure 12 shows

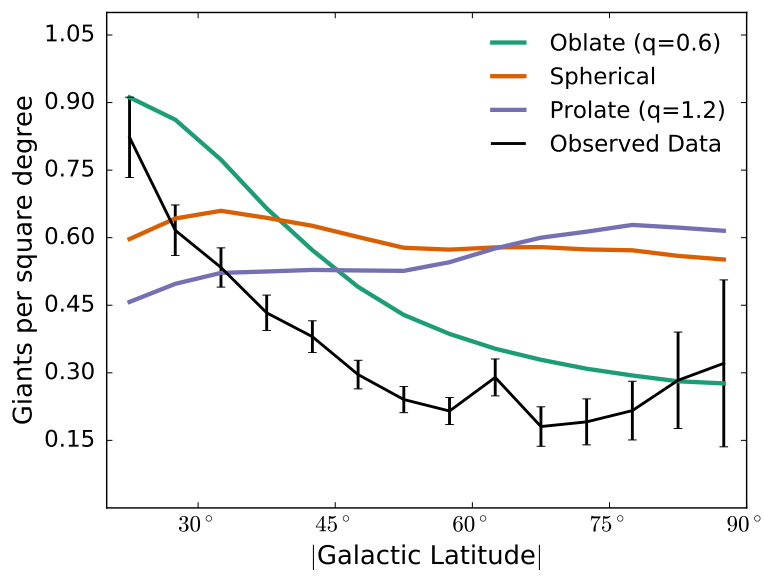

Figure 12. Halo shape models after removing all fields within $12^{\circ}$ of the Sagittarius stream. The models are not renormalized to fit to the subselected data. This behavior of this subset of the data is more consistent with an oblate halo, suggesting by comparison with Figure 11 that the Sagittarius stream was responsible for an overdensity of stars at high latitude.

the same plot of the density with Galactic latitude, but where all fields within $12^{\circ}$ of the Sagittarius plane have been excluded. The remaining fields are broadly consistent with a flattened halo, and shows a significantly reduced density at high latitude compared to the full sample.

One could be tempted to consider a measurement with Sagittarius excluded as more representative of the halo, but this implies an unphysical distinction between massive structures of tidal debris that are recognizable and 
the rest of the substructured halo which does not appear as individually recognizable features. We do not report a fit of the Sagittarius-removed data for this reason. A summary statistic of the halo shape that accounts for and captures the prevalence of substructure would be a much preferable treatment, but this is a complex task which we leave to a subsequent work.

\section{DISCUSSION}

The stellar halo beyond $30-40 \mathrm{kpc}$ is a difficult region to study; tracers bright enough to be found robustly at the depth of current large-scale surveys are rare and can be difficult to distinguish from nearby contaminants. The result of this is that studies have typically used either RR Lyrae or BHB stars for this region where mainsequence stars are not readily accessible.

Studies of RR Lyrae have reported steep slopes of $\sim-4$ to -5 beyond $20-30 \mathrm{kpc}$, e.g., -4.5 by Watkins et al. (2009). The region of sensitivity is comparable to our work, yet significantly discrepant from our measurement of -3.5 . The origin of this disagreement is unclear, but we can consider a few possible sources. The Watkins et al. (2009) work uses RR Lyrae from the SDSS stripe 82, which covers $290 \mathrm{deg}^{2}$. It is possible that slopes measured in relatively small patches of the sky will be highly variable due to the substructured nature of the halo, as surveys may happen upon underdense or overdense regions. This is independent of the tracer used, and can only be alleviated with larger surveys.

A survey of BHB stars in the 2dF Quasar Redshift Survey by De Propris et al. (2010) over a $750 \mathrm{deg}^{2}$ area and with data extending out to $90 \mathrm{kpc}$ found a much shallower slope of -2.5 . A much smaller survey used deep observations of $\mathrm{F}$ stars over a $23 \mathrm{deg}^{2}$ field to obtain a slope of -4.85 to $60 \mathrm{kpc}$ (Pila-Díez et al. 2015). The same caveat of substructure applies in these surveys as well; we expect large variations in halo densities measured by smaller surveys. We note that our sample may be similarly affected, if at a lesser degree due to the larger area coverage. The substructured halo will cause variation between surveys above and beyond Poissonian uncertainties.

One of the larger samples of halo stars beyond $30 \mathrm{kpc}$ comes from Deason et al. (2014), who used a photometric selection process to identify BHB stars in the SDSS imaging footprint. While this does alleviate much of the issues with smaller surveys, the photometric selection of BHB stars is a particularly fraught process. We note that at magnitudes needed to probe the outer halo with BHB stars the number of contaminant blue stragglers and quasars substantially exceeds the number of target BHB stars (see Fig. 8 of Deason et al. 2014); this is the case for most tracers of the outer halo. The separation in broadband color between the target BHB population and the contaminant population is, however, only 0.09 magnitudes according to the loci assumed by Deason et al. (2014), though the intrinsic widths of the distributions, prior to any photometric error, are 0.04 magnitudes. This separation becomes even more difficult in the presence of the photometric uncertainties at $g \sim 19$. The lack of decisive separation between the populations presents a tremendous challenge to any attempts at extracting the individual densities, and makes the resulting density profile particularly sensitive to any discrepancies between the chosen models and the behavior of the actual populations.

We argue that our data have a much clearer separation between the target tracer population and any contaminants, though at the cost of much additional telescope time in obtaining the narrowband data. Our work significantly disagrees with the finding of a halo slope of -6 beyond $50 \mathrm{kpc}$ by Deason et al. (2014). Such a steep profile would require essentially zero giants to exist between 18th and 19th magnitude in our survey, and less than half of the number that we detect between 17 th and 18th magnitudes (see the middle panels of Figure 3).

Another program that has sought to characterize the halo profile is presented in Xue et al. (2015), where SEGUE spectroscopy provides the additional information for separating bright giant tracers from contaminants. Of their fitted models, their broken power law model is most similar to ours since our data start beyond their break radius, and they find a power law slope of $-3.8 \pm 0.1$. This is similar to our result, though slightly steeper. We note though that this measurement is primarily driven by data inside of $\sim 50 \mathrm{kpc}$; beyond this distance giants become rare in the SEGUE selection (a total of seven stars beyond $65 \mathrm{kpc}$ ) and Poisson noise becomes significant (See Figure 7, Xue et al. 2015). Even with this small number of stars, Xue et al. (2015) find their data to be formally inconsistent with the steep drop beyond $50 \mathrm{kpc}$ suggested by Deason et al. (2014). Further confirmation of this comes from the RR Lyrae profile of Cohen et al. (2015), who find a profile consistent with $r^{-3.8}$ between 50 and $100 \mathrm{kpc}$. Their sample is similar to the spectroscopic giant measurements of Xue et al. (2015) in that the sample is extremely clean, but with the risk of incompleteness at large distances which must be corrected statistically.

\section{CONCLUSIONS}

Our results show an extensive and unbroken halo beyond $\sim 30 \mathrm{kpc}$ out to $80-90 \mathrm{kpc}$, with a power law profile of -3.5 . Our ability to measure the profile robustly at these large distances is a result of bringing additional decisive information beyond broadband photometry, enabling a strong separation between the intrinsically bright tracers of interest and the more numer- 
ous dwarf star contamination. Our data cover a large area of sky to significant depth, further mitigating the common problems of sparse sampling and biases towards nearby regions.

While knowledge of our own Galaxy is of interest on its own, our derived Milky Way halo density profile will be particularly informative in comparison with both other galaxies and with simulations and models of stellar halo formation in general. We are only recently able to start putting our own outer halo in context with those of a number of nearby galaxies, e.g., NGC 253 (Bailin et al. 2011; Greggio et al. 2014), M 101 (van Dokkum et al. 2014), Andromeda (Courteau et al. 2011; Ibata et al. 2014), and the several galaxies in the GHOSTS survey (Monachesi et al. 2015). These comparisons will give us an observational understanding of the degree to which the Milky Way's halo is "average" or an outlier, and will begin to tells us about the accretion history of the Milky Way in general. This is part of what motivates our focus on the entire halo rather than removing "substructure"; this substructure (or lack thereof) is likely what carries the information on the accretion history that we are most interested in. Comparing the bulk properties of the halo, rather than the properties after removing substructure, is the only level basis for comparison we will have between the Milky Way and external galaxies. Any identification and removal of individual structures is closely tied to the specific types of observational data available, and the character of available data is vastly different between external galaxies and our own.

Pursuing these comparisons in depth will require further work, both observational and theoretical. Theoretically, we must develop a robust understanding of the connection between a stellar halo profile and its underlying dark matter halo and accretion history. This will require high fidelity simulations of diverse halo assembly histories, though this is a challenging area for simulations (Bailin et al. 2014). Observationally, a full comparison between halos will require metrics that can account for and measure substructure quantitatively; this will be the subject of a subsequent work.

This work was partially supported by NSF grant AST 1008342. D.L.N. was supported by a McLaughlin Fellowship at the University of Michigan. S.R.M. was supported by NSF grant 1413269 .

Funding for SDSS-III has been provided by the Alfred P. Sloan Foundation, the Participating Institutions, the National Science Foundation, and the U.S. Department of Energy Office of Science. The SDSS-III web site is http://www.sdss3.org/. SDSS-III is managed by the Astrophysical Research Consortium for the Participating Institutions of the SDSS-III Collaboration including the University of Arizona, the Brazilian Participation Group, Brookhaven National Laboratory, Carnegie Mellon University, University of Florida, the French Participation Group, the German Participation Group, Harvard University, the Instituto de Astrofisica de Canarias, the Michigan State/Notre Dame/JINA Participation Group, Johns Hopkins University, Lawrence Berkeley National Laboratory, Max Planck Institute for Astrophysics, Max Planck Institute for Extraterrestrial Physics, New Mexico State University, New York University, Ohio State University, Pennsylvania State University, University of Portsmouth, Princeton University, the Spanish Participation Group, University of Tokyo, University of Utah, Vanderbilt University, University of Virginia, University of Washington, and Yale University.

\section{REFERENCES}

Akhter, S., Da Costa, G. S., Keller, S. C., \& Schmidt, B. P. 2012, ApJ, 756, 23

An, D., Beers, T. C., Johnson, J. A., et al. 2013, ApJ, 763, 65

Bailin, J., Bell, E. F., Chappell, S. N., Radburn-Smith, D. J., \& de Jong, R. S. 2011, ApJ, 736, 24

Bailin, J., Bell, E. F., Valluri, M., et al. 2014, ApJ, 783, 95

Bell, E. F., Zucker, D. B., Belokurov, V., et al. 2008, ApJ, 680, 295

Belokurov, V., Zucker, D. B., Evans, N. W., et al. 2006, ApJL, 642, L137

Bertin, E., \& Arnouts, S. 1996, A\&AS, 117, 393

Clark, J. P. A., \& McClure, R. D. 1979, PASP, 91, 507

Cohen, J. G., Sesar, B., Banholzer, S., \& PTF Consortium, t. 2015, arXiv:1509.05997

Courteau, S., Widrow, L. M., McDonald, M., et al. 2011, ApJ, 739, 20

Deason, A. J., Belokurov, V., \& Evans, N. W. 2011, MNRAS, 416, 2903

Deason, A. J., Belokurov, V., Evans, N. W., \& Johnston, K. V. 2013, ApJ, 763, 113
Deason, A. J., Belokurov, V., Koposov, S. E., \& Rockosi, C. M. 2014, ApJ, 787, 30

de Jong, J. T. A., Yanny, B., Rix, H.-W., et al. 2010, ApJ, 714, 663

De Propris, R., Harrison, C. D., \& Mares, P. J. 2010, ApJ, 719, 1582

Dotter, A., Chaboyer, B., Jevremović, D., et al. 2008, ApJS, 178, 89

Geisler, D. 1984, PASP, 96, 723

Greggio, L., Rejkuba, M., Gonzalez, O. A., et al. 2014, A\&A, 562, A73

Ibata, R. A., Lewis, G. F., McConnachie, A. W., et al. 2014, ApJ, 780, 128

Ivezić, Ž., Sesar, B., Jurić, M., et al. 2008, ApJ, 684, 287

Jurić, M., Ivezić, Ž., Brooks, A., et al. 2008, ApJ, 673, 864

Font, A. S., McCarthy, I. G., Crain, R. A., et al. 2011, MNRAS, 416,2802

Foreman-Mackey, D., Hogg, D. W., Lang, D., \& Goodman, J. 2013, PASP, 125, 306

Kroupa, P. 2001, MNRAS, 322, 231 
Majewski, S. R., Ostheimer, J. C., Kunkel, W. E., \& Patterson, R. J. 2000, AJ, 120, 2550

Majewski, S. R., Skrutskie, M. F., Weinberg, M. D., \& Ostheimer, J. C. 2003, ApJ, 599, 1082

Monachesi, A., Bell, E. F., Radburn-Smith, D., et al. 2015, arXiv:1507.06657

Pila-Díez, B., de Jong, J. T. A., Kuijken, K., van der Burg, R. F. J., \& Hoekstra, H. 2015, A\&A, 579, A38

Purcell, C. W., Bullock, J. S., \& Kazantzidis, S. 2010, MNRAS, 404, 1711

Robin, A. C., Reylé, C., Derrière, S., \& Picaud, S. 2003, A\&A, 409, 523

Sesar, B., Ivezić, Ž., Grammer, S. H., et al. 2010, ApJ, 708, 717

Sesar, B., Jurić, M., \& Ivezić, Ž. 2011, ApJ, 731, 4

Slater, C. T., Bell, E. F., Schlafly, E. F., et al. 2014, ApJ, 791, 9
Starkenburg, E., Helmi, A., Morrison, H. L., et al. 2009, ApJ, 698, 567

Stetson, P. B. 1987, PASP, 99, 191

Tody, D. 1986, Proc. SPIE, 627, 733

Tody, D. 1993, Astronomical Data Analysis Software and Systems II, 52, 173

van Dokkum, P. G., Abraham, R., \& Merritt, A. 2014, ApJL, 782, L24

Watkins, L. L., Evans, N. W., Belokurov, V., et al. 2009, MNRAS, 398, 1757

Xue, X.-X., Rix, H.-W., Yanny, B., et al. 2011, ApJ, 738, 79

Xue, X.-X., Rix, H.-W., Ma, Z., et al. 2015, ApJ, 809, 144

Zasowski, G., Johnson, J. A., Frinchaboy, P. M., et al. 2013, AJ, 146,81

Zolotov, A., Willman, B., Brooks, A. M., et al. 2009, ApJ, 702, 1058 